\title{
The Role of Low-Energy $(\leq 20 \mathrm{eV})$ Electrons in Astrochemistry
}

\author{
Michael C. Boyer, ${ }^{1}$ Nathalie Rivas, ${ }^{2}$ Audrey A. Tran, ${ }^{2}$ Clarissa A. Verish, ${ }^{2}$ and Christopher R. \\ Arumainayagam, ${ }^{\mathrm{a}, 2}$ \\ ${ }^{1}$ Department of Physics, \\ Clark University, \\ Worcester, MA 01610 \\ ${ }^{2}$ Department of Chemistry, \\ Wellesley College, \\ Wellesley, MA 02481
}

\begin{abstract}
UV photon-driven condensed phase cosmic ice reactions have been the main focus in understanding the extraterrestrial synthesis of complex organic molecules. Low-energy $(\leq 20 \mathrm{eV})$ electron-induced reactions, on the other hand, have been largely ignored. In this article, we review studies employing surface science techniques to study low-energy electron-induced condensed phase reactions relevant to astrochemistry. In particular, we show that low-energy electron irradiation of methanol ices leads to the synthesis of many of the same complex molecules formed through UV irradiation. Moreover, our results are qualitatively consistent with the hypothesis that high-energy condensed phase radiolysis is mediated by low-energy electroninduced reactions. In addition, due to the numbers of available low-energy secondary electrons resulting from the interaction of high-energy radiation with matter as well as differences between electron- and photon-induced processes, low-energy electron-induced reactions are perhaps as, or even more, effective than photon-induced reactions in initiating condensed-phase chemical reactions in the interstellar medium. Consequently, we illustrate a need for astrochemical models to include the details of electron-induced reactions in addition to those driven by UV photons. Finally, we show that low-energy electron-induced reactions may lead to the production of unique molecular species that could serve as tracer molecules for electron-induced condensed phase reactions in the interstellar medium.
\end{abstract}

\footnotetext{
${ }^{a}$ Corresponding Author: Telephone: 781-283-3326; FAX: 781-283-3642; Email: carumain@ wellesley.edu
} 


\section{Introduction}

\subsection{Complex Molecules in Space}

Ever since the discovery of the still enigmatic spectral diffuse interstellar bands (DIB) about 80 years ago,[1] we have known that space is filled with complex molecules.[2] In addition to these optical spectral absorption bands, vibrational emission bands have been used to telescopically identify complex molecules such as polyaromatic hydrocarbons (PAH), fullerenes $\left(\mathrm{C}_{60}, \mathrm{C}_{70}\right)$, and diamondoids.[3] Moreover, (sub)millimeter rotational transitions of molecules have been exploited to identify within interstellar and circumstellar clouds approximately 200 different gas phase molecules including glycolaldehyde $\left(\mathrm{HOCH}_{2} \mathrm{CHO}\right),[4]$ a potential prebiotic molecule. The synthesis of such complex/prebiotic molecules in the interstellar medium is thought to occur via three possible mechanisms: (1) gas-phase reactions, (2) surface reactions on bare carbonaceous or silicaceous dust grains, and (3) energetic processing of $\sim 100 \mathrm{ML}$ (monolayer)-thick ice mantles surrounding micron-sized dust grains.[5] In this review, we will explore the use of surface science techniques to understand the third mechanism, energetic ice processing, which includes both surface and bulk reactions. Specifically, we will review recent work which examines the role of low-energy electrons in the synthesis of prebiotic molecules in analogs of methanol cosmic (interstellar, planetary, and cometary) ices.

\subsection{UV Photon-Induced Ice Chemistry}

In recent years, laboratory experiments and theoretical calculations have suggested that UV photon-induced surface and bulk processing of icy grain mantles containing water, carbon monoxide, methanol, and ammonia is one of the main mechanisms for the synthesis of complex organic molecules found in hot molecular cores and corinos, the regions within dark, dense 
molecular clouds warmed by one or more nearby protostars.[6],[7],[8] The source of UV light that initiates these chemical reactions is believed to be local because externally sourced UV radiation cannot penetrate these clouds. UV light emission from hydrogen molecules excited by cosmic rays[9] is thought to photoprocess icy grain mantles found in these dark, dense molecular clouds, [10] leading to the production of both light (e.g., $\bullet \mathrm{H})$ and heavy (e.g., $\mathrm{CH}_{3} \mathrm{O} \bullet$ ) radicals. While diffusion of light radicals is possible near $10 \mathrm{~K},[11]$ the gradual warm-up from $\sim 10 \mathrm{~K}$ to $100 \mathrm{~K}$ in hot cores and hot corinos allows for the diffusion of heavier radicals. Low temperature, barrier-less, radical-radical reactions may then result in the synthesis of complex molecules, such as methyl formate $\left(\mathrm{HCOOCH}_{3}\right)$, which are potential precursors of biological molecules.[12] Building upon early investigations,[6],[13] recent laboratory experiments that simulate photochemistry within methanol ices in the interstellar medium have shown the formation of organic molecules such as acetaldehyde $\left(\mathrm{CH}_{3} \mathrm{CHO}\right)$, glycolaldehyde $\left(\mathrm{HOCH}_{2} \mathrm{CHO}\right)$, methyl formate $\left(\mathrm{HCOOCH}_{3}\right)$, formic acid $(\mathrm{HCOOH})$, acetic acid $\left(\mathrm{CH}_{3} \mathrm{COOH}\right)$, ethylene glycol $\left(\left(\mathrm{CH}_{2} \mathrm{OH}\right)_{2}\right)$, dimethyl ether $\left(\mathrm{CH}_{3} \mathrm{OCH}_{3}\right)$, and ethanol $\left(\mathrm{CH}_{3} \mathrm{CH}_{2} \mathrm{OH}\right)$ following $\mathrm{UV}$ photolysis of condensed methanol.[14] In their 2009 paper, the authors accordingly concluded that "models show that photochemistry in ices followed by desorption may explain the observed abundances" of gas-phase complex molecules detected in hot cores.[14] However, very recent observational work studying relative abundances of methanol and other complex organic molecules (COMs) near low-mass protostars concluded that "astrochemical models still underpredict the abundances of key complex organic molecules, such as methyl formate or dimethyl ether, suggesting that our understanding of their formation remains incomplete."[15] 


\subsection{Low-Energy Electron-Induced Ice Reactions}

In addition to being initiated by UV light, extraterrestrial ice reactions may also be triggered by cosmic ray-induced, non-thermal, low-energy, secondary electrons, whose effects have been largely ignored in previous experimental and theoretical astrochemistry studies. Interactions of high-energy radiation (e.g., cosmic rays, $\gamma$-rays, $\mathrm{x}$-rays, electrons, and ions) with matter produce enormous numbers $\left(\sim 4 \times 10^{4}\right.$ electrons per MeV of energy deposited $)$ of nonthermal secondary low-energy electrons.[16] While secondary products such as excited species and ions produced from the high-energy radiation interacting with molecules cause some radiation damage, it is the secondary electron-molecule inelastic collisions that are thought to be the primary driving forces in a wide variety of radiation-induced chemical reactions.[17],18] 


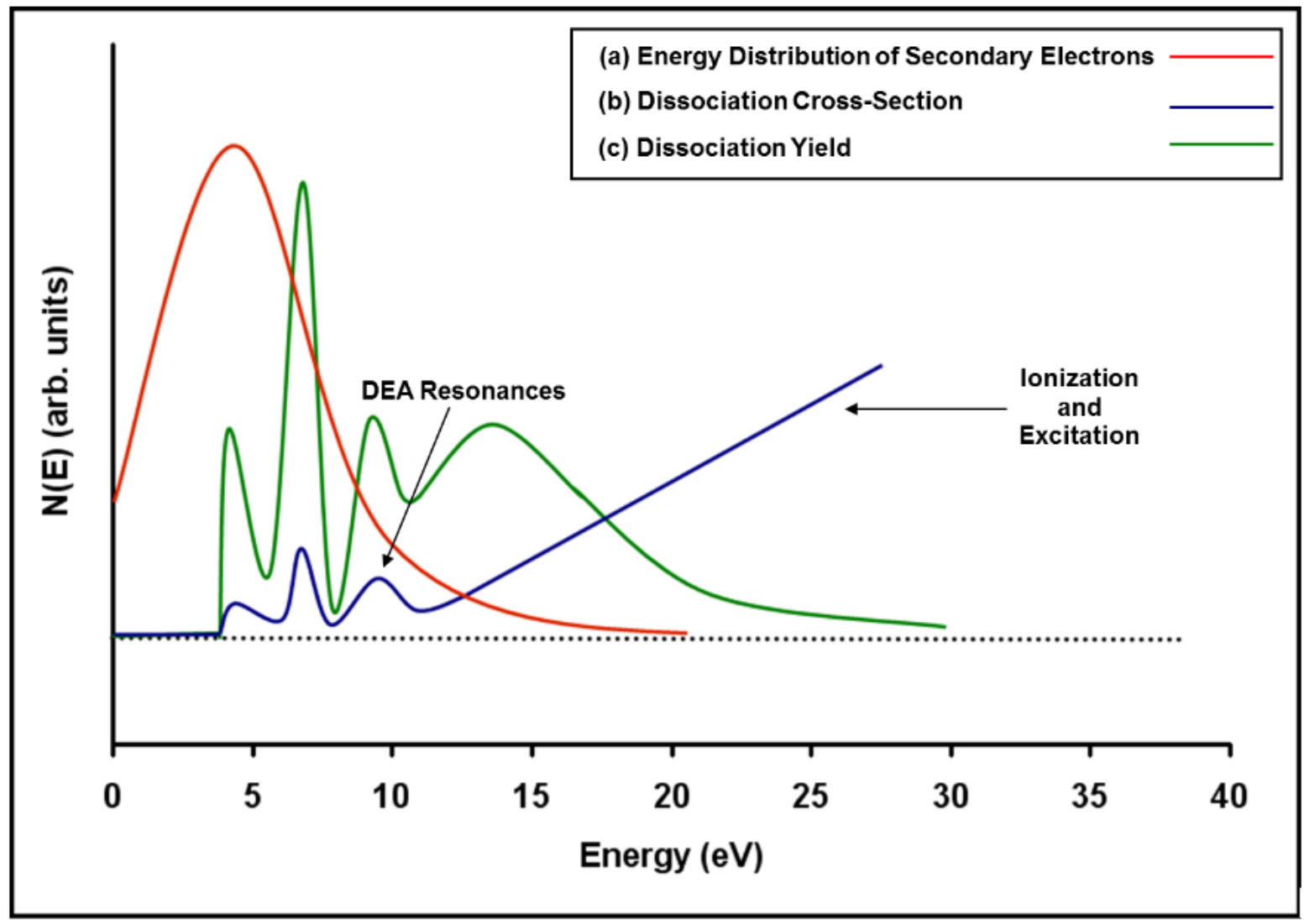

Figure 1

Schematic of (a) energy distribution of secondary electrons generated during a primary ionizing event; (b) cross-section for electron-induced dissociation for a typical molecule; (c) dissociation yield as a function of electron energy for a typical molecule. Reproduced from a previous publication.[19]

As shown schematically in Figure 1(a), secondary electrons resulting from the interactions of high-energy radiation with matter typically have energies below $\sim 20 \mathrm{eV}$. Figure 1(b) illustrates the dissociation cross-section of a generic molecule as a function of incident electron energy. Featured prominently in this plot are resonance peaks characteristic of dissociative electron attachment (DEA), a resonant process occurring at low electron energies $(<$ $15 \mathrm{eV})$. Electron-impact excitation and electron-impact ionization processes typically occur at 
energies above $\sim 3$ and $\sim 10 \mathrm{eV}$ respectively, and have dissociation cross-sections which typically increase monotonically with electron energy. The molecular dissociation yield as a function of electron energy (Figure 1(c)) is the result of multiplying the molecular dissociation cross-section (Figure 1(b)) by the energy distribution of the secondary electrons (Figure 1(a)). Hence, even though the dissociation probability increases with increasing electron energy, the dissociation yield is expected to be greatest at low electron energies $(<15 \mathrm{eV})$ due to the abundance of secondary electrons at those energies (Figure 1).

We hypothesize that cosmic ray-induced low-energy electron processing of interstellar ices and dust may occur via three mechanisms: (1) the interaction of cosmic rays with gaseous molecular hydrogen produces low-energy electrons that can interact with the surface molecules of cosmic ices and submonolayer adsorbates on dust grains, (2) the interaction of cosmic rays with molecules within ices generates a cascade of low-energy electrons which interact with ice surface molecules and those contained within the bulk of the ice mantles, and (3) the interactions

of the cosmic rays with the dust grain cores beneath the ice mantle generate low-energy electrons that can interact with molecules at the bottom ice layers in contact with the dust grains.[20]

\subsection{Electron-Molecule Interactions: A Brief Introduction}

Electron-induced dissociation generally occurs via ionization, excitation, and attachment processes:[19]

(a) Electron-impact ionization of a generic molecule $\mathrm{AB}$ to yield $\mathrm{AB}^{+*}$, an excited state cation, occurs at incident electron energies above $\sim 10 \mathrm{eV}$ and may be characterized by the following equation:

$$
\mathrm{e}^{-}+\mathrm{AB} \rightarrow \mathrm{AB}^{+*}+2 \mathrm{e}^{-}
$$

The ionized parent molecule may undergo ion-molecule reactions or fragment: 


$$
\mathrm{AB}^{+*} \rightarrow \mathrm{A}+\mathrm{B}^{+^{*}}
$$

(b) Electron-impact excitation occurs at incident electron energies above $\sim 3 \mathrm{eV}$ :

$$
\mathrm{e}^{-}+\mathrm{AB} \rightarrow \mathrm{AB}^{*}+\mathrm{e}^{-}
$$

If the excited neutral molecule is not in a bound state, $\mathrm{AB}^{*}$ may dissociate:

$$
\mathrm{AB}^{*} \rightarrow \cdot \mathrm{A}^{*}+\cdot \mathrm{B}
$$

Or, dissociation of the excited molecule may occur via another channel, dipolar dissociation (DD), following electron-impact excitation. In this process the resultant excited electronic state induces ion-pair formation:

$$
\mathrm{AB}^{*} \rightarrow \mathrm{A}^{+}+\mathrm{B}^{-}
$$

DD is delineated by a continuous increase in the radiolysis product yield above $\sim 10-15 \mathrm{eV}$.

(c) Electron attachment to form a temporary negative ion ${ }^{\mathrm{b}}$ (TNI) typically occurs at electron energies below $15 \mathrm{eV}$ and is characterized by the following equation:

$$
\mathrm{e}^{-}+\mathrm{AB} \rightarrow \mathrm{AB}^{-*}
$$

The formation of a temporary negative ion is a resonant process because the final state $\left(\mathrm{AB}^{-*}\right)$ is a discrete state. Dissociative electron attachment (DEA) occurs when the temporary negative ion undergoes bond scission, resulting in an anion $\left(\mathrm{B}^{-}\right)$in addition to a neutral atom/radical $(\mathrm{A} \cdot)$ :

$$
\mathrm{AB}^{-*} \rightarrow \cdot \mathrm{A}+\mathrm{B}^{-}
$$

In a yield vs. electron energy plot (Figure 1(c)), DEA is typically characterized by resonances below $\sim 20 \mathrm{eV}$.

\subsection{Photon- vs. Electron-Induced Ice Chemistry}

Because of similarities between photon- and electron-induced excitations/ionizations, both vacuum ultraviolet (VUV) photons $(6-12 \mathrm{eV})$ and low-energy $(<20 \mathrm{eV})$ electrons will

\footnotetext{
${ }^{\mathrm{b}}$ The lifetime of a temporary negative ion ranges from $10^{-15}$ to $10^{-2} \mathrm{~s}$.
} 
drive similar chemistry, though yields and product ratios will likely differ. Due to selection rules, governed primarily by dipole interactions and spin conservation, photon-molecule interactions are more restrictive compared to electron-molecule interactions. For example, unlike for electrons,[21] photon-induced singlet-to-triplet transitions are nominally forbidden. Furthermore, in contrast to photons, electrons can be captured into the aforementioned resonant temporary negative ion (TNI) states which subsequently may dissociate.[19],[22] The resulting molecular fragments may then react with the parent molecule or other daughter products to yield products unique to electron irradiation. In other words, due to differences in how electrons and photons interact with molecules, low-energy electron-molecule collisions, a fundamental step resulting from the processing of cosmic ices by high-energy radiation, could theoretically lead to the synthesis of molecules not accessible via UV photochemistry. There is experimental evidence which provides some support for this claim.[23],[24],[25]

In addition to the synthesis of unique molecular species, low-energy, electron-induced chemistry may predominate over UV photon-induced chemistry. For example, reaction crosssections can be several orders of magnitude larger for electrons than for photons, particularly at incident energies corresponding to resonances associated with DEA. Such a dominance of electron-induced over photon-induced condensed-phase chemical reactions has already been documented for a few molecular species.[26],[27],[28] Moreover, because the chemistry ascribed to photon irradiation in thin films adsorbed on surfaces may be due to film- and substrategenerated photoelectrons, the role of electrons compared to photons may be underestimated in comparative studies. 


\section{Low-Energy Electron-Induced Reactions of Methanol Ices}

Methanol, found in relatively high abundances in cosmic ices, is not only believed to be an important precursor to species such as methyl formate $\left(\mathrm{HCOOCH}_{3}\right)$ and dimethyl ether $\left(\mathrm{CH}_{3} \mathrm{OCH}_{3}\right)$, but also to many prebiotic species such as simple sugars and amino acids.[29],[30],[31] Numerous studies of the high-energy radiolysis of methanol have been conducted during the past seven decades.[32],[33],[34],[35],[36] Photolysis of condensed methanol has been studied using post-irradiation analysis.[37],[38],[39] Electron-stimulated desorption experiments have been used to study the interactions of low-energy electrons with condensed methanol.[40],[41],[42] While several post-irradiation studies of low-energy electroninduced reactions of condensed $\mathrm{CH}_{3} \mathrm{OH}$ have also been carried out elsewhere,[43], [44] the focus of this review will be experiments almost exclusively conducted at Wellesley College.[42],[45],[46],[47]

\subsection{Technical Approach for Ice Chemistry Experiments}

Electron-induced methanol ice experiments were performed at Wellesley College in a custom-designed stainless steel UHV chamber with a base pressure of $5 \times 10^{-10}$ Torr. The Mo(110) single crystal sample was cooled to $\sim 90 \mathrm{~K}$ with liquid nitrogen and heated to $\sim 800 \mathrm{~K}$ radiatively or to $\sim 2200 \mathrm{~K}$ by electron bombardment. Direct dosers with precision leak valves allowed for controlled deposition of methanol onto the clean $\operatorname{Mo}(110)$ crystal surface at $\sim 90 \mathrm{~K}$ to obtain multilayer films of sufficient thickness ( $\geq 10$ monolayers) to minimize the influence of the metal on the outermost adsorbate layers. Electron irradiation of the condensed thin films was accomplished using a low-energy flood electron gun (Kimball Physics model FRA-2X1-2) capable of producing quasi-monoenergetic $( \pm 0.4 \mathrm{eV})$ electrons of desired energy in the range of $2 \mathrm{eV}$ to $1000 \mathrm{eV}$. Following electron irradiation, temperature programmed desorption (TPD) 
experiments were conducted to identify radiolysis/photolysis products using a triple filtered Hiden Ion Desorption Probe (IDP) Series 500 quadrupole mass spectrometer. As the surface was heated from $100 \mathrm{~K}$ to $700 \mathrm{~K}$ via radiative heating at $\sim 7 \mathrm{~K} / \mathrm{s}$, temperature programmed desorption data were collected with the mass spectrometer and the thermocouple, both of which were interfaced to a computer. In addition, post-irradiation infrared reflection absorption spectroscopy measurements in the absence of surface heating were performed using a TENSORTM 27 FTIR spectrometer (Bruker Optics) equipped with a liquid nitrogen-cooled mercury cadmium telluride (MCT) detector.[47]

\subsection{Identification of low-energy electron-induced reaction products of methanol}

In our first radiation chemistry study, temperature programmed desorption and electronstimulated desorption were successfully employed to probe the molecular fragmentation caused by low-energy electrons incident on multilayer thin films of methanol.[45] We identified, for the first time, methoxymethanol $\left(\mathrm{CH}_{3} \mathrm{OCH}_{2} \mathrm{OH}\right)$, a highly labile species, as a reaction product of electron-induced dissociation of methanol.[45] We have conducted three further studies investigating the low-energy $(\leq 20 \mathrm{eV})$ electron-induced reactions of condensed methanol.[42],[46],[47] As described in more detail below, using temperature programmed desorption and infrared reflection absorption spectroscopy, we have identified fifteen electroninduced methanol radiolysis products, many of which have been previously identified as being formed by methanol UV photolysis in the interstellar medium.

\subsubsection{Post-Irradiation Temperature Programmed Desorption of Methanol}

Figure 2 shows temperature programmed desorption data following irradiation of 20 monolayers of methanol by $20 \mathrm{eV}$ electrons for 20 minutes at an incident current of $2 \mu \mathrm{A}$ (flux $\approx$ $2 \times 10^{13}$ electrons $/ \mathrm{cm}^{2} / \mathrm{s}$ and fluence $\approx 3 \times 10^{16}$ electrons $\left./ \mathrm{cm}^{2}\right)$. The temperature programmed 
desorption data show desorption features which allow us to identify eleven low-energy electroninduced radiolysis products of methanol. ${ }^{\mathrm{c}}$ The identifications of these electron-induced methanol radiolysis products were based on (1) comparison to known mass spectra, (2) temperature programmed desorption data for methanol films containing the suspected radiolysis product, (3) results of analogous experiments with methanol isotopologues $\left({ }^{13} \mathrm{CH}_{3} \mathrm{OH}\right.$ and $\left.\mathrm{CD}_{3} \mathrm{OD}\right)$, and (4) trends in boiling points and desorption temperatures.[46]

Of these eleven radiolysis products identified following $20 \mathrm{eV}$ electron irradiation of condensed methanol,[46] formaldehyde $\left(\mathrm{H}_{2} \mathrm{CO}\right)$, dimethyl ether $\left(\mathrm{CH}_{3} \mathrm{OCH}_{3}\right)$, methyl formate $\left(\mathrm{HCOOCH}_{3}\right)$, acetaldehyde $\left(\mathrm{CH}_{3} \mathrm{CHO}\right)$, glycolaldehyde $\left(\mathrm{HOCH}_{2} \mathrm{CHO}\right)$, acetic acid $\left(\mathrm{CH}_{3} \mathrm{COOH}\right)$, ethanol $\left(\mathrm{CH}_{3} \mathrm{CH}_{2} \mathrm{OH}\right)$, and ethylene glycol $\left(\left(\mathrm{CH}_{2} \mathrm{OH}\right)_{2}\right)$ have also been identified as products following UV photon irradiation of condensed methanol.[14] Furthermore, results of similar temperature programmed desorption studies conducted following lower-energy $(7 \mathrm{eV})$ electronirradiation of 20 monolayers of $\mathrm{CH}_{3} \mathrm{OH}$ demonstrated the formation of the same radiolysis products except for acetaldehyde, glycolic acid $\left(\mathrm{HOCH}_{2} \mathrm{CO}_{2} \mathrm{H}\right)$, and 1,2,3-propanetriol $\left(\mathrm{HOCH}_{2} \mathrm{CHOHCH}_{2} \mathrm{OH}\right)$.[46] We should note that glycolic acid and 1,2,3-propanetriol, which are identified in Figure 2, are not believed to be nascent primary radiolysis products of methanol.[46] These experimental results suggest that low-energy, electron-induced condensed phase reactions may also contribute to the interstellar synthesis of complex molecules whose synthesis was previously attributed to UV photolysis of cosmic ices.

Using temperature programmed desorption experiments, we have identified all of the abovementioned $20 \mathrm{eV}$ electron-induced radiolysis products, except acetaldehyde, following higher-energy $(1000 \mathrm{eV})$ electron irradiation of methanol ices.[47] If we ignore this slight

\footnotetext{
${ }^{\mathrm{c}}$ As described in section 2.1.2, four other methanol radiolysis products were identified using post-irradiation infrared reflection absorption spectroscopy.
} 
discrepancy, attributable to the challenges associated with analysing a complex mixture with fifteen or more species, these findings are qualitatively consistent with the hypothesis that highenergy condensed phase radiolysis is mediated by low-energy electron-induced reactions.[47]

We have identified methoxymethanol $\left(\mathrm{CH}_{3} \mathrm{OCH}_{2} \mathrm{OH}\right)$ as an electron-induced product following irradiation of condensed methanol with 7, 20, 55, and $1000 \mathrm{eV}$ electrons. [42],[45],[46],[47] Methoxymethanol has also been recently identified using reflectron time-offlight mass spectrometry (ReTOF) following irradiation of condensed methanol with $5000 \mathrm{eV}$ electrons.[48] Methoxymethanol, however, has not been identified as a UV photolysis product of condensed methanol.[6],[13],[14] This discrepancy suggests differences in electron- versus UV photon-induced condensed phase chemistry.

Experiments involving isotopic labeling of different methanol layers revealed that the constituent atoms of the radiolysis products originate exclusively in the methanol multilayers upon electron irradiation.[45] Independent temperature programmed desorption experiments of ethylene glycol and methanol on clean $\mathrm{Mo}(110)$ in the absence of electron irradiation demonstrated that multilayers of ethylene glycol desorb at the same temperature at which ethylene glycol desorbs following methanol irradiation.[45] These experimental results demonstrate that these radical-radical reactions occur in the multilayers and not in the monolayer. 


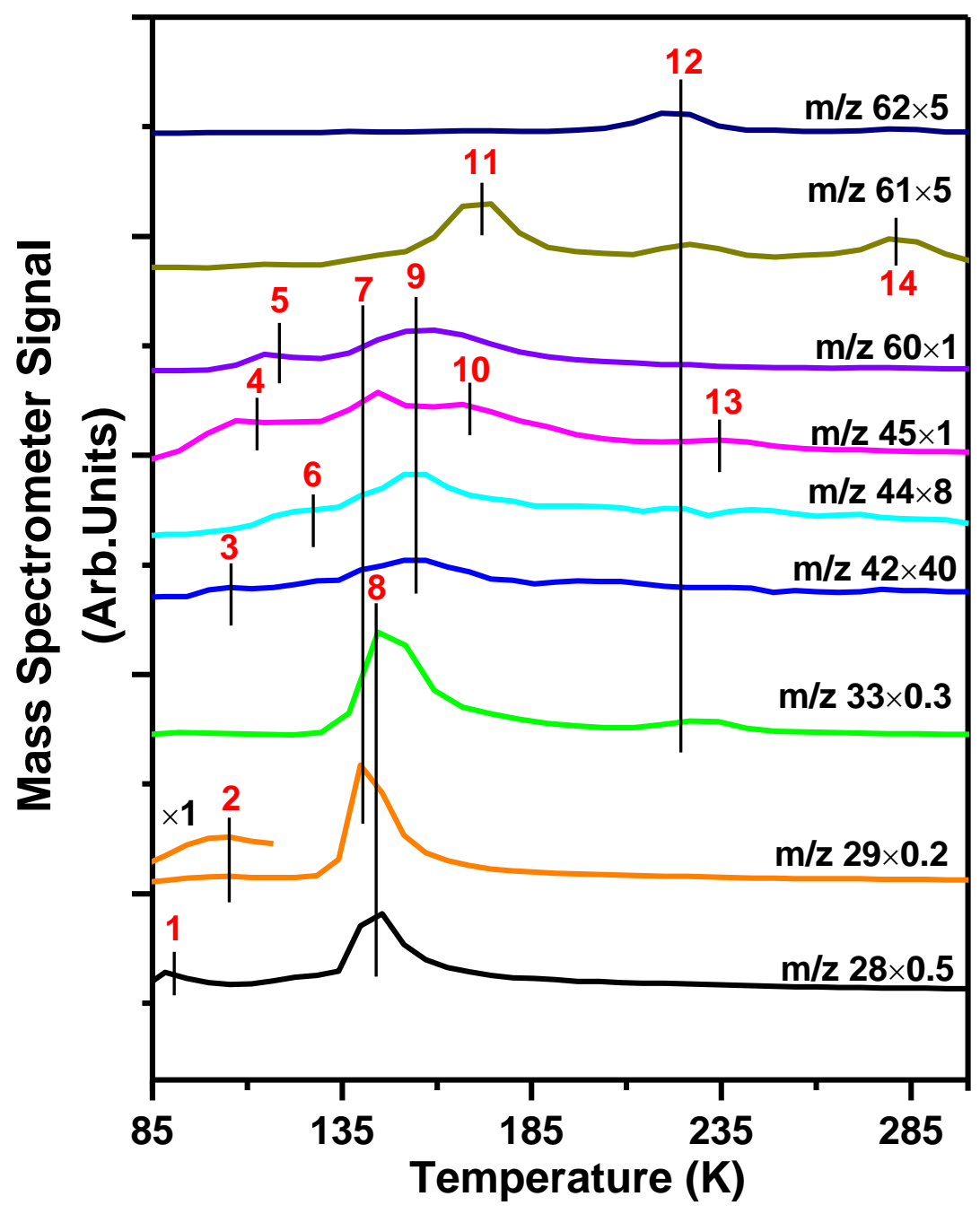

Figure 2

Post-irradiation temperature programmed desorption data for 20 monolayers of ${ }^{12} \mathrm{CH}_{3} \mathrm{OH}$ irradiated with $20 \mathrm{eV}$ electrons for 20 minutes at an incident current of $2 \mu \mathrm{A}$ (flux $\approx 2 \times$ $10^{13}$ electrons $/ \mathrm{cm}^{2} / \mathrm{s}$ and fluence $\approx 3 \times 10^{16}$ electrons $/ \mathrm{cm}^{2}$ ) shows several desorption features: (1) $\mathrm{CO}$ (background), (2) formaldehyde $\left(\mathrm{H}_{2} \mathrm{CO}\right)$, (3) unknown, (4) dimethyl ether $\left(\mathrm{CH}_{3} \mathrm{OCH}_{3}\right)$, (5) methyl formate $\left(\mathrm{HCOOCH}_{3}\right)$, (6) acetaldehyde $\left(\mathrm{CH}_{3} \mathrm{CHO}\right)$, (7) glycolaldehyde $\left(\mathrm{HOCH}_{2} \mathrm{CHO}\right)$, (8) methanol $\left(\mathrm{CH}_{3} \mathrm{OH}\right)$, (9) acetic acid $\left(\mathrm{CH}_{3} \mathrm{COOH}\right),(10)$ ethanol $\left(\mathrm{CH}_{3} \mathrm{CH}_{2} \mathrm{OH}\right)$, (11) methoxymethanol $\left(\mathrm{CH}_{3} \mathrm{OCH}_{2} \mathrm{OH}\right)$, (12) ethylene glycol $\left(\left(\mathrm{CH}_{2} \mathrm{OH}\right)_{2}\right)$, (13) glycolic acid $\left(\mathrm{HOCH}_{2} \mathrm{CO}_{2} \mathrm{H}\right),(14)$ 1,2,3-propanetriol $\left(\mathrm{HOCH}_{2} \mathrm{CHOHCH}_{2} \mathrm{OH}\right)$. Plots vertically offset for clarity. We note that methanol has a 
natural abundance of ${ }^{13} \mathrm{C}$ (about $1 \%$ ) which allows us to monitor $\mathrm{m} / \mathrm{z}=33$ to detect

methanol in our experiments. Reproduced from a previous publication.[46]

\subsubsection{Post-Irradiation Infrared Reflection Absorption Spectroscopy}

While temperature programmed desorption experiments require thermal processing to detect and identify products as they desorb from the irradiated thin films, infrared spectroscopy does not require heating of the film/surface for product identification. We used post-irradiation infrared reflection absorption spectroscopy to study low-energy (14 eV) and higher-energy (900 eV) electron-irradiated condensed films of methanol 90 K.[47] In addition to identifying ethylene glycol, formaldehyde, and dimethyl ether, products previously identified through temperature programmed desorption experiments, we detected via infrared spectroscopy four additional methanol radiolysis products: methane $\left(\mathrm{CH}_{4}\right)$, carbon dioxide $\left(\mathrm{CO}_{2}\right)$, carbon monoxide (CO), and the hydroxyl methyl radical $\left(\bullet \mathrm{CH}_{2} \mathrm{OH}\right)$. Figure 3 illustrates the infrared spectroscopic identification of four $\left(\left(\mathrm{CH}_{2} \mathrm{OH}\right)_{2}, \mathrm{H}_{2} \mathrm{CO}, \mathrm{CH}_{3} \mathrm{OCH}_{3}\right.$, and $\left.\bullet \mathrm{CH}_{2} \mathrm{OH}\right)$ of the above mentioned seven radiolysis products. Given that infrared reflection absorption spectroscopy is less sensitive than mass spectrometry, it is not surprising that clear infrared signatures were not found for all products identified through temperature programmed desorption experiments. Nevertheless, our infrared spectroscopy findings are also qualitatively consistent with the hypothesis that highenergy condensed phase radiolysis is mediated by low-energy electron-induced reactions.[47] Using a combination of mass spectrometry and infrared spectroscopy, we have identified a total of fifteen electron-induced methanol radiolysis products, demonstrating the clear benefit of using complementary surface science techniques to study energetic processing of cosmic ice analogs. 


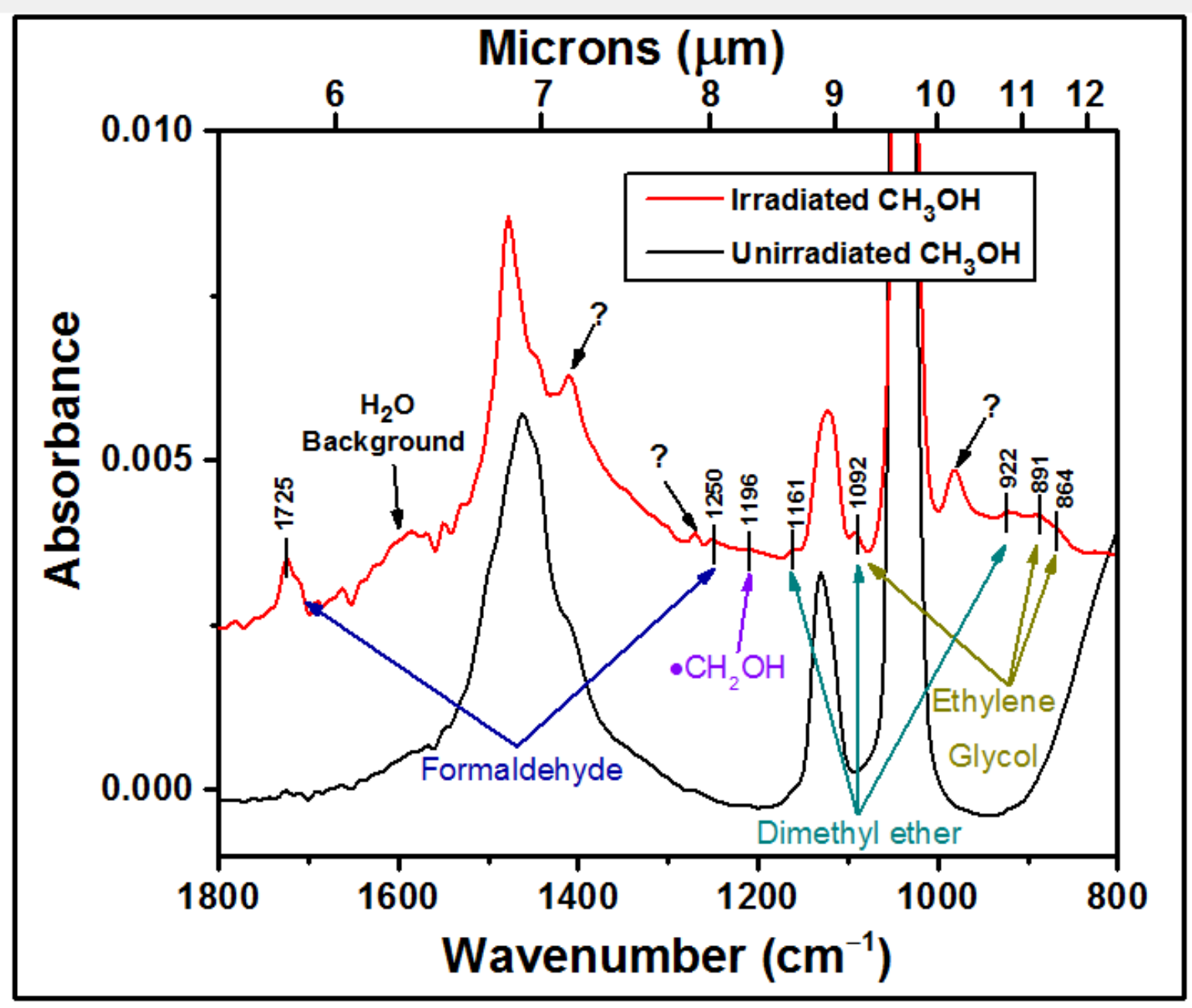

Figure 3

Infrared reflection absorption spectroscopy of $100 \mathrm{ML}$ of unirradiated (black curve) and irradiated (14 eV electrons for 20 minutes at a transmitted current of $2 \mu \mathrm{A}$ ) (red curve) condensed $\mathrm{CH}_{3} \mathrm{OH}$. The hydroxymethyl radical $\left(\cdot \mathrm{CH}_{2} \mathrm{OH}\right)$ can be identified by a single weak peak at $1196 \mathrm{~cm}^{-1}$ in the irradiated $\mathrm{CH}_{3} \mathrm{OH}$ curve. This peak is difficult to observe due to the figure scaling. There are a number of yet to be identified peaks indicated by "?"s. Reproduced from a previous publication.[47] 


\subsection{Dynamics of Low-Energy Electron-Induced Reactions}

Based on (1) our temperature programmed desorption results which demonstrate the formation of eleven methanol products following irradiation by $7 \mathrm{eV}$ electrons and (2) the secondary electron energy distribution which peaks below $\sim 15 \mathrm{eV}$, we suggest that processes other than electron-impact ionization may dominate ionizing radiation-induced chemical synthesis in environments such as the interstellar medium. To further investigate the dynamics of low-energy electron induced reactions in condensed films of methanol, we conducted temperature programmed desorption and electron-stimulated desorption (ESD) experiments as a function of electron irradiation energy.

\subsubsection{Radiolysis Yield vs. Incident Electron Energy}

For further study, we selected two methanol radiolysis products: ethylene glycol $\left(\mathrm{HOCH}_{2} \mathrm{CH}_{2} \mathrm{OH}\right)$ and methoxymethanol $\left(\mathrm{CH}_{3} \mathrm{OCH}_{2} \mathrm{OH}\right)$.[42] The dependence of the reaction cross section on initial electron energy for each of these two products was investigated to obtain additional information regarding the dynamics of electron-induced dissociation. Post-irradiation temperature programmed desorption experiments were used to determine the yield of ethylene glycol and methoxymethanol as a function of incident electron energy while keeping constant the film thickness, incident electron current, and irradiation time (Figure 4).[42] The minimum incident electron energies for the formation of ethylene glycol and methoxymethanol were found to be $\sim 5 \mathrm{eV}$ and between 6 and $7 \mathrm{eV}$, respectively. ${ }^{\mathrm{d}}$ However, the total yield of ethylene glycol and methoxymethanol evinced no obvious resonant structures, suggesting that non-resonant

\footnotetext{
${ }^{\mathrm{d}}$ We note that while we did monitor ethylene glycol yield as a function of incident electron energy (Figure 4(b)), the threshold energy for ethylene glycol formation from irradiated methanol ices was determined by monitoring ethene (Figure 4(c)). Ethene is produced as the result of a surface reaction between ethylene glycol and the Mo(110) substrate. [45] Therefore, ethene formation must be monitored to fully understand the dynamics of ethylene glycol formation.
} 
electron-impact excitation is the primary mechanism by which electrons initiate ethylene glycol and methoxymethanol formation below the ionization threshold.

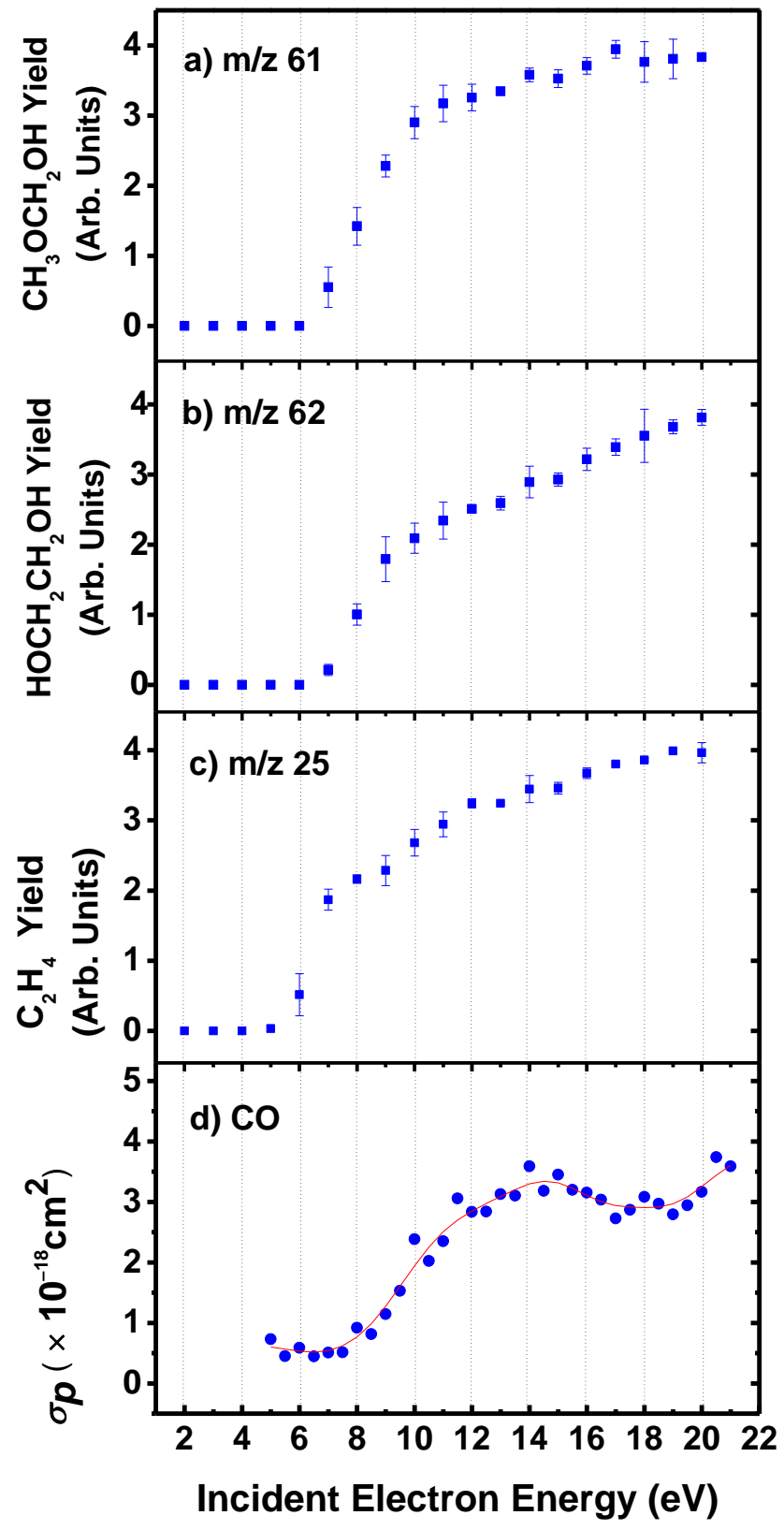

Figure 4

Methanol thin films of 10 and 15 ML thickness were irradiated by electrons with energies ranging from $2 \mathrm{eV}$ to $20 \mathrm{eV}$. During these experiments, the electron dose rate was $\sim 1.3$ $\times 10^{13}$ electron/s (clean crystal current of $2 \mu \mathrm{A}$ ) with a total dose of $\sim 1.1 \times 10^{16}$ electrons (15 minutes irradiation time). The top three plots, methoxymethanol yield (a), ethylene 
glycol yield (b), ethene yield (c) as a function of incident electron energy, are the result of averaging three trials (for $\mathrm{m} / \mathrm{z}=61$ and 62) and two trials (for $\mathrm{m} / \mathrm{z}=25$ ) for each electron energy. Plot (d) shows the $\mathrm{CO}$ production cross section as a function of incident electron energy. Reproduced from a previous publication.[42]

\subsubsection{Radiolysis Yield vs. Electron Fluence}

For ethylene glycol and methoxymethanol, the dependence of the yield on electron fluence (electron flux multiplied by exposure time) was probed. As shown in Figure 5, the radiolysis product yields were found to increase quadratically with increasing electron fluence below a fluence of $3 \times 10^{12} \mathrm{~cm}^{-2}$.[42] Because this finding is consistent with the radiolysis products being formed from products of two independent dissociation events, a mechanism involving radicalradical reactions was proposed:[42]

$$
\begin{gathered}
\mathrm{CH}_{3} \mathrm{OH} \stackrel{e^{-}}{\longrightarrow}\left[\mathrm{CH}_{3} \mathrm{OH}\right]^{*}+e^{-} \text {(8) } \\
{\left[\mathrm{CH}_{3} \mathrm{OH}\right]^{*} \longrightarrow \mathrm{CH}_{2} \mathrm{OH}+\cdot \mathrm{H}^{*} \text { (9) }} \\
{\left[\mathrm{CH}_{3} \mathrm{OH}\right]^{*} \longrightarrow \mathrm{CH}_{3} \mathrm{O}+\cdot \mathrm{H} \quad \text { (10) }} \\
\mathrm{CH}_{3} \mathrm{O}+\mathrm{CH}_{2} \mathrm{OH} \longrightarrow \mathrm{CH}_{3} \mathrm{OCH}_{2} \mathrm{OH} \text { (11) } \\
{\left[\mathrm{CH}_{2} \mathrm{OH}+\left[\mathrm{CH}_{2} \mathrm{OH} \longrightarrow \mathrm{HOCH}_{2} \mathrm{CH}_{2} \mathrm{OH}(12)\right.\right.}
\end{gathered}
$$




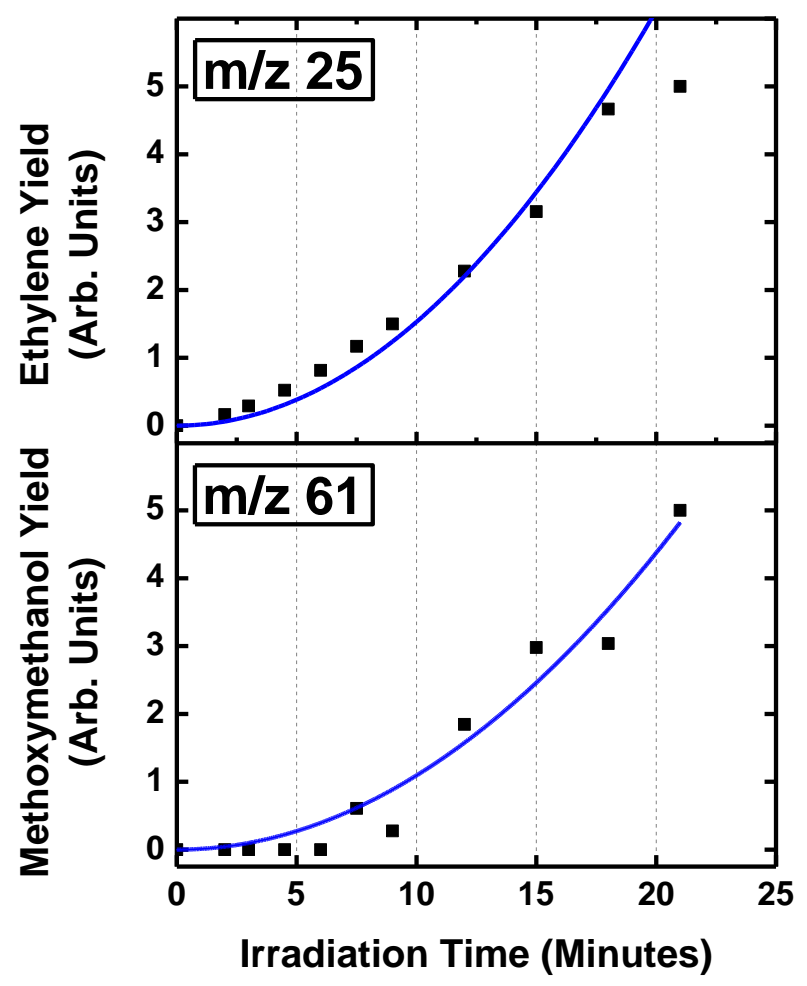

Figure 5

Product yield as a function of fluence showing the yield of ethene (ethylene glycol) determined by monitoring $\mathrm{m} / \mathrm{z}=25$ and the yield of methoxymethanol determined by monitoring $m / z=61$. In these experiments, methanol films of constant thickness were irradiated by $8.0 \mathrm{eV}$ electrons. The electron dose rate (flux) in each experiment was $\sim 1.3$ $\times 10^{12}$ electrons $/ \mathrm{cm}^{2} / \mathrm{s}$ with a total dose (fluence) ranging from $\sim 1.6 \times 10^{15}$ to $\sim 1.6 \times 10^{16}$ electrons $/ \mathrm{cm}^{2}$ ( 2 to 21 minutes irradiation time). Reproduced from a previous publication.[42] 


\subsubsection{Electron-Induced Isothermal Desorption}

Our results of electron-stimulated desorption experiments, conducted at the University of Sherbrooke and involving a high-sensitivity time-of-flight mass spectrometer, indicate that anion $\left(\mathrm{H}^{-}, \mathrm{CH}^{-}, \mathrm{CH}_{2}{ }^{-}, \mathrm{CH}_{3}{ }^{-}, \mathrm{O}^{-}, \mathrm{OH}^{-}\right.$, and $\left.\mathrm{CH}_{3} \mathrm{O}^{-}\right)$desorption from the methanol thin film at incident electron energies below about $15 \mathrm{eV}$ is dominated by processes initiated by the dissociation of temporary negative ions of methanol formed via electron capture (Figure 6).[42] This finding is particularly surprising since our ethylene glycol/methoxymethanol radiolysis yield vs. electron energy graph does not evince resonances attributable to dissociative electron attachment. We attribute this apparent dichotomy between the results of electron-stimulated desorption and postirradiation temperature programmed desorption experiments to methanol's low dissociative electron attachment cross section. 


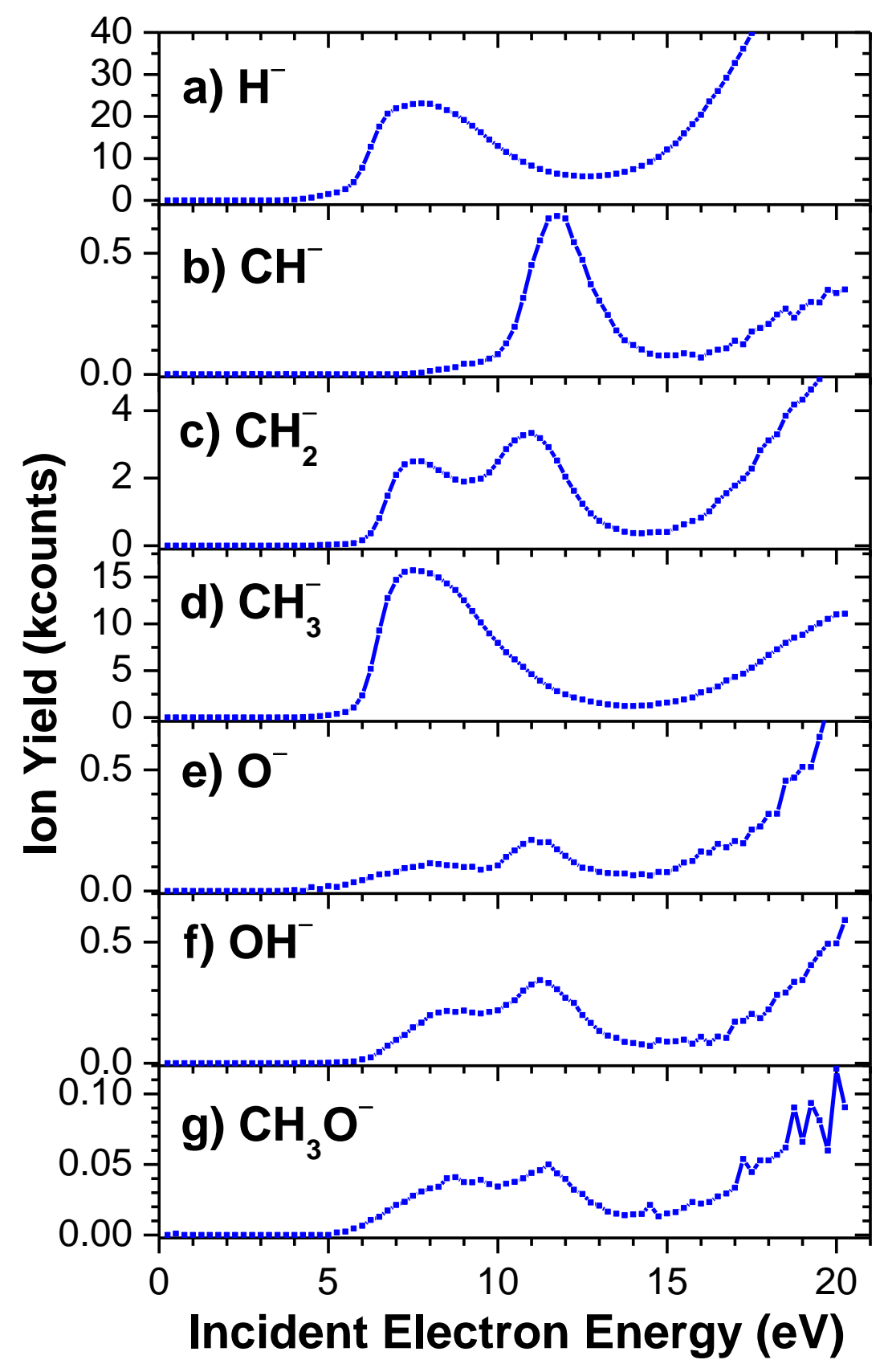

Figure 6

Mass spectrometer signal as a function of incident electron energy for the different anions desorbing during electron-irradiation. Four monolayer thick methanol films cooled to 35 $\mathrm{K}$ were irradiated by electrons $(0-20 \mathrm{eV})$ using a Kimball Physics ELG-2 electron gun with a resolution of $0.5 \mathrm{eV}$. Reproduced from a previous publication.[42] 


\section{The Role of Low-Energy Electrons in Astrochemistry}

In the gas phase, based on the relative intensities of atomic emission lines of $\mathrm{H}, \mathrm{O}$, and $\mathrm{C}$ from within the coma of comet 67P/Churyumov-Gerasimenko, a very recent study has attributed the rapid breakup of $\mathrm{H}_{2} \mathrm{O}$ and $\mathrm{CO}_{2}$ to low-energy electrons with peak energies in the range of 25 to $50 \mathrm{eV}$.[49] In the condensed phase, results of Monte Carlo simulations of high-energy radiation, specifically $20 \mathrm{keV}$ electrons, interacting with water demonstrate that nearly $90 \%$ of the secondary and tertiary electrons have initial energies less than $20 \mathrm{eV}$.[50] Given that resonances for dissociative electron attachment typically occur at energies below $20 \mathrm{eV}$ with very high cross sections, it is crucial to understand the role of very low-energy electrons in condensed phase astrochemistry. Low-energy electrons may result from two processes: (1) the interaction of cosmic rays with gaseous molecular hydrogen present in the dark, dense molecular clouds, ${ }^{e}$ and (2) the inelastic collisions that the cosmic ray experiences as it traverses through the ice-covered dust grains. Although other high-energy radiolysis secondary products lead to some radiolytic changes, the inelastic collisions of the low-energy electrons with matter are hypothesized to be the primary driving force in a wide variety of radiation-induced chemical reactions.[51] Our experiments have investigated the processing of astrochemically-relevant laboratory ices by low-energy $(\leq 20 \mathrm{eV})$ electrons characteristic of the secondary electrons produced by the interactions of high-energy radiation, such as cosmic rays, with matter. We have identified fifteen low-energy electron-induced methanol radiolysis products, many of which have been previously identified as forming via methanol UV photolysis in the interstellar medium (Table 1). Coupled with the results of our higher-energy electron experiments, our findings are

\footnotetext{
${ }^{\mathrm{e}}$ These low-energy electrons will interact with only the top few layers of cosmic ices; our experiments simulate these interactions.
} 
qualitatively consistent with the hypothesis that high-energy condensed phase radiolysis is mediated by low-energy electron-induced reactions. Furthermore, our studies suggest that electron-induced reactions of methanol relevant to the synthesis of complex molecules in the interstellar medium are likely driven predominantly through electron-impact excitation rather than electron-impact ionization or dissociative electron attachment.

\begin{tabular}{|c|c|c|c|c|}
\hline & $\begin{array}{c}\text { This Work } \\
{[42] \cdot[46][[47]}\end{array}$ & $\begin{array}{c}\text { Allamandola et al. } \\
{[13]}\end{array}$ & $\begin{array}{c}\text { Oberg et al. } \\
\text { [14] }\end{array}$ & $\begin{array}{c}\text { Gerakines et al. } \\
{[6]}\end{array}$ \\
\hline & $\begin{array}{l}\text { Electrons } \\
(\leq 20 \mathrm{eV})\end{array}$ & UV & UV & UV \\
\hline$\bullet \mathrm{CH}_{2} \mathrm{OH}$ & $\sqrt{ }$ & & $\sqrt{ }$ & $\sqrt{ }$ \\
\hline $\mathrm{H}_{2} \mathrm{CO}$ & $\sqrt{ }$ & $\sqrt{ }$ & $\sqrt{ }$ & $\sqrt{ }$ \\
\hline HCO & & $\sqrt{ }$ & $\sqrt{ }$ & $\sqrt{ }$ \\
\hline $\mathrm{CO}$ & $\sqrt{ }$ & $\sqrt{ }$ & $\sqrt{ }$ & $\sqrt{ }$ \\
\hline $\mathrm{CO}_{2}$ & $\sqrt{ }$ & $\sqrt{ }$ & $\sqrt{ }$ & $\sqrt{ }$ \\
\hline $\mathrm{CH}_{4}$ & $\sqrt{ }$ & $\sqrt{ }$ & $\sqrt{ }$ & $\sqrt{ }$ \\
\hline НСООН & & & $\sqrt{ }$ & \\
\hline $\mathrm{CH}_{3} \mathrm{OCHO}$ & $\sqrt{ }$ & & $\sqrt{ }$ & $\sqrt{ }$ \\
\hline $\mathrm{CH}_{3} \mathrm{OCH}_{3}$ & $\sqrt{ }$ & & $\sqrt{ }$ & \\
\hline $\mathrm{HOCH}_{2} \mathrm{CH}_{2} \mathrm{OH}$ & $\sqrt{ }$ & & $\sqrt{ }$ & $\sqrt{ }$ \\
\hline $\mathrm{CH}_{3} \mathrm{OCH}_{2} \mathrm{OH}$ & $\sqrt{ }$ & & & \\
\hline $\mathrm{HCOCH}_{2} \mathrm{OH}$ & $\sqrt{ }$ & & $\sqrt{ }$ & \\
\hline $\mathrm{CH}_{3} \mathrm{CH}_{2} \mathrm{OH}$ & $\sqrt{ }$ & & $\sqrt{ }$ & \\
\hline $\mathrm{C}_{2} \mathrm{H}_{6}$ & & & $\sqrt{ }$ & \\
\hline $\mathrm{CH}_{3} \mathrm{CHO}$ & $\sqrt{ }$ & & $\sqrt{ }$ & \\
\hline $\mathrm{CH}_{3} \mathrm{COOH}$ & $\sqrt{ }$ & & $\sqrt{ }$ & \\
\hline $\mathrm{HOCH}_{2} \mathrm{CO}_{2} \mathrm{H}$ & $\sqrt{ }$ & & & \\
\hline $\mathrm{HOCH}_{2} \mathrm{CHOHCH}_{2} \mathrm{OH}$ & $\sqrt{ }$ & & & \\
\hline
\end{tabular}

Table 1: Comparison of several studies which investigated the condensed phase reactions of $\mathrm{CH}_{3} \mathrm{OH}$ initiated by low-energy electrons and $\mathrm{UV}$ photons. The identification of the last two species in the table was tentative.

While our post-irradiation temperature programmed desorption data clearly demonstrate the formation of methoxymethanol following irradiation of methanol with electrons with energies as low as $6 \mathrm{eV}$, previous studies[37],[38],[39] have not reported methoxymethanol as a photolysis product of methanol. If methoxymethanol can be shown to form only via electron 
irradiation of methanol, this molecule could serve as a tracer molecule for electron-induced reactions in the interstellar medium. ${ }^{\mathrm{f}}$

Most comparisons between photon- and electron-induced condensed phase reactions have involved studies conducted in different laboratories with different equipment and different techniques. A few studies have employed the same experimental protocols to probe the differences between condensed phase reactions induced by electrons and photons.[23],[25],[26],[27],[28] Nevertheless, even these very limited investigations have involved electrons/photons whose energies exceed the ionization threshold. Such high-energy electrons/photons produce a low-energy electron cascade, precluding direct comparison between electron- and photon-induced condensed phase reactions. The use of sub-ionization electrons/photons in our future experiments will avoid the production of low-energy secondary electrons.

Finally, what occurs in the laboratory and in space must also be modelled using computational techniques such as the kinetic (stochastic) Monte Carlo method and the continuous time random walk approach.[52],[53] Irradiation of ice grains in space by cosmic radiation results in the production of a cascade of low-energy electrons with energies in the range of 0 to $20 \mathrm{eV}$. It will be challenging to simulate how these electrons are thermalized in the ice grain, losing energy to the medium and producing a variety of different chemically reactive ions and radicals in a spatially nonhomogeneous track.

Given that astrochemically-relevant electron-induced chemistry is still in its nascent phase, a quantitative discussion and comparison is not yet possible for reactions initiated by electrons vs. photons. For example, estimations of secondary electron-fluxes require

\footnotetext{
${ }^{\mathrm{f}}$ To the best of our knowledge, methoxymethanol has not yet been identified spectroscopically in the interstellar medium.
} 
sophisticated modeling. ${ }^{\mathrm{g}}$ Furthermore, a comparison of molecular dissociation cross-sections between UV photons and electrons cannot yet be made. Ultimately, taking a comparison of UV vs. electron fluxes in the interstellar medium in conjunction with UV vs. electron molecular dissociation cross-sections will allow for a true comparison between the relative importance of electron and UV-induced chemistry. Our radiolysis studies of condensed methanol films suggest a need for the inclusion of low-energy electron-driven processes in astrochemical models to obtain a more complete understanding of synthesis mechanisms and abundances of complex organic molecules in space.

\section{Conclusions}

We have performed the first systematic study of the dynamics of low-energy $(\leq 20 \mathrm{eV})$ electron-induced condensed matter reactions relevant to astrophysical phenomena. Such experiments complemented by future low-energy photolysis studies and stochastic Monte Carlo simulations will provide a quantitative understanding of the relative roles of electrons and photons in the synthesis of prebiotic molecules in cosmic ices and interstellar dust grains found near star-forming regions of the universe. Such studies may ultimately help us better understand the initial stages of the genesis of life.

\section{Acknowledgements}

CRA acknowledges with much gratitude the sage mentorship by Professor John Yates since 1995. The first methanol paper was written while CRA was on sabbatical leave working with John. This work was supported by grants from the National Science Foundation (NSF grant

\footnotetext{
${ }^{g}$ Professor Simon Pimblott personal communications: "Stochastic Monte Carlo methods have been developed to model the attenuation of low energy electrons in water ice predicting both the spatial location and the nature of the energy transfer events. Spatially resolved track structures give information about the physico-chemical processes occurring, such as ionization, excitation, and dissociative electron attachment and recombination as well as electron thermalization and hydration. Track structure simulation provides a spatial distribution of reactive radiation induced species that can be used to model the diffusion kinetic radiation chemistry of the system using either a random flights or an independent reaction times approach."
} 
number CHE-1012674 and CHE-1465161) and Wellesley College (Faculty awards and Brachman Hoffman small grants). We thank Dr. Andrew D. Bass and Professor Léon Sanche for invaluable assistance. 


\section{$\underline{\text { References }}$}

[1] P.W. Merrill, Astrophysical Journal 83 (1936) 126-128.

[2] G.H. Herbig, Annual Review of Astronomy and Astrophysics 33 (1995) 19-73.

[3] A. Tielens, Reviews of Modern Physics 85 (2013) 1021-1081.

[4] J.M. Hollis, F.J. Lovas, P.R. Jewell, Astrophysical Journal 540 (2000) L107-L110.

[5] E. Herbst, Physical Chemistry Chemical Physics 16 (2014) 3344-3359.

[6] P.A. Gerakines, W.A. Schutte, P. Ehrenfreund, Astronomy and Astrophysics 312 (1996) 289-305.

[7] D. Williams, S. Viti, Philosophical Transactions of the Royal Society a-Mathematical Physical and Engineering Sciences 371 (2013) 1-12.

[8] E. Herbst, E.F. van Dishoeck, in: R. Blandford, J. Kormendy, E. VanDishoeck (Eds.), Annual Review of Astronomy and Astrophysics, Vol 47, 2009, pp. 427-480.

[9] S.S. Prasad, S.P. Tarafdar, Astrophysical Journal 267 (1983) 603-609.

[10] R. Gredel, S. Lepp, A. Dalgarno, E. Herbst, Astrophysical Journal 347 (1989) 289-293.

[11] N. Watanabe, Y. Kimura, A. Kouchi, T. Chigai, T. Hama, V. Pirronello, Astrophysical Journal Letters 714 (2010) L233-L237.

[12] R.T. Garrod, E. Herbst, Astronomy \& Astrophysics 457 (2006) 927-936.

[13] L.J. Allamandola, S.A. Sandford, G.J. Valero, Icarus 76 (1988) 225-52.

[14] K.I. Oberg, R.T. Garrod, E.F. van Dishoeck, H. Linnartz, Astronomy \& Astrophysics 504 (2009) 891U28.

[15] V. Taquet, A. Lopez-Sepulcre, C. Ceccarelli, R. Neri, C. Kahane, S.B. Charnley, Astrophysical Journal 804 (2015).

[16] I.G. Kaplan, A.M. Miterev, Adv. Chem. Phys. 68 (1987) 255-386.

[17] A.D. Bass, L. Sanche, Low Temp. Phys. (Translation of Fizika Nizkikh Temperatur (Kiev)) 29 (2003) 202-214.

[18] N.J. Mason, AIP Conference Proceedings 680 (2003) 885.

[19] C.R. Arumainayagam, H.L. Lee, R.B. Nelson, D.R. Haines, R.P. Gunawardane, Surface Science Reports 65 (2010) 1-44.

[20] Cuppen, et al., Faraday Discussions 168 (2014) 571-615.

[21] K.H. Becker, C.W. McCurdy, T.M. Orlando, T.N. Rescigno, Electron-Driven Processes: Scientific Challenges and Technological Opportunities, 2000.

[22] E. Alizadeh, L. Sanche, Chemical Reviews 112 (2012) 5578-5602.

[23] X.L. Zhou, J.M. White, Journal of Chemical Physics 92 (1990) 5612-5621.

[24] R.L. Hudson, M.H. Moore, Astrophysical Journal 568 (2002) 1095-1099.

[25] S.C. Sparks, A. Szabo, G.J. Szulczewski, K. Junker, J.M. White, Journal of Physical Chemistry B 101 (1997) 8315-8323.

[26] M.A. Henderson, R.D. Ramsier, J.T. Yates, Journal of Vacuum Science \& Technology a-Vacuum Surfaces and Films 9 (1991) 1563-1568.

[27] T.B. Scoggins, H. Ihm, Y.M. Sun, J.M. White, Journal of Physical Chemistry B 103 (1999) 6791-6802.

[28] E. Alizadeh, P. Cloutier, D. Hunting, L. Sanche, Journal of Physical Chemistry B 115 (2011) 45234531.

[29] L. Allamandola, D. Hudgins, in: V. Pirronello, J. Krelowski, G. Manicò (Eds.), Solid State Astrochemistry, Springer Netherlands, 2003, pp. 251-316.

[30] D.P.P. Andrade, Boechat-Roberty, H. M., Martinez, R., Homem, M. G. P., da Silveira, E. F., Rocco, M. L. M., Surface Science 603 (2009) 1190-1196.

[31] J.M. Hollis, Lovas, F.J., Jewell, P.R., The Astrophysical Journal 540 (2000) 107-110. 
[32] W.J. Skraba, J.G. Burr, Jr., D.N. Hess, J. Chem. Phys. 21 (1953) 1296-1296.

[33] G.G. Odian, A. Rossi, E.N. Trachtenberg, Journal of Polymer Science 42 (1960) 575-8.

[34] A.B. Dempster, Zerbi, G. , J. Chem. Phys 54 (1971) 3600-\&.

[35] R.I. Kaiser, Bennet, C.J., Chen, S., Sun, B., Chang, A.H.H., The Astrophysical Journal 660 (2007) 1588-1608.

[36] Y.J. Chen, A. Ciaravella, G.M.M. Caro, C. Cecchi-Pestellini, A. Jimenez-Escobar, K.J. Juang, T.S. Yih, Astrophysical Journal 778 (2013) 162 1-11.

[37] L.J. Allamandola, Sandford, S. A., Valero, G. J., Icarus 76 (1988) 225-252.

[38] K.I. Oberg, Garrod, R.T., van Dishoeck, E.F., Linnartz, H. , A\&A 504 (2009) 819-913.

[39] P.A. Gerakines, Schutte, W. A., Ehrenfreund, P., A\&A 312 (1996) 289.

[40] L. Parenteau, J.P. Jay-Gerin, L. Sanche, J. Phys. Chem. 98 (1994) 10277-81.

[41] A.T. Wen, M. Michaud, L. Sanche, Phys. Rev. A: At., Mol., Opt. Phys. 54 (1996) 4162-4170.

[42] M.C. Boyer, M.D. Boamah, K.K. Sullivan, C.R. Arumainayagam, M. Bazin, A.D. Bass, L. Sanche, The Journal of Physical Chemistry C 118 (2014) 22592-22600.

[43] J.M. White, Schwaner, A.L., J. Phys. Chem B 101 (1997) 10414-10422.

[44] M. Lepage, M. Michaud, L. Sanche, J. Chem. Phys. 107 (1997) 3478-3484.

[45] T.D. Harris, D.H. Lee, M.Q. Blumberg, C.R. Arumainayagam, J. Phys. Chem. 99 (1995) 9530-5.

[46] M.D. Boamah, K.K. Sullivan, K.E. Shulenberger, C.M. Soe, L.M. Jacob, F.C. Yhee, K.E. Atkinson, M.C. Boyer, D.R. Haines, C.R. Arumainayagam, Faraday Discussions 168 (2014) 249-266.

[47] Kristal K. Sullivan, Mavis D. Boamah, Katie E. Shulenberger, Sitara Chapman, Karen E. Atkinson, Michael C. Boyer, C.R. Arumainayagam, Monthly Notices of the Royal Astronomical Society (Submitted).

[48] S. Maity, R.I. Kaiser, B.M. Jones, Physical Chemistry Chemical Physics 17 (2015) 3081-3114.

[49] P.D. Feldman, et al., Astronomy \& Astrophysics 583 (2015).

[50] V. Cobut, Y. Frongillo, J.P. Patau, T. Goulet, M.J. Fraser, J.P. Jay-Gerin, Radiat. Phys. Chem. 51 (1998) 229-243.

[51] N.J. Mason, AIP Conference Proceedings 680 (2003) 885-888.

[52] Q. Chang, H.M. Cuppen, E. Herbst, Astronomy \& Astrophysics 434 (2005) 599-611.

[53] R.T. Garrod, Astrophysical Journal 778 (2013). 


\title{
Formation of secondary electrons in cosmic ices and dust grains
}

\author{
secondary electron cascade
}

$$
\text { o-20 eV }
$$

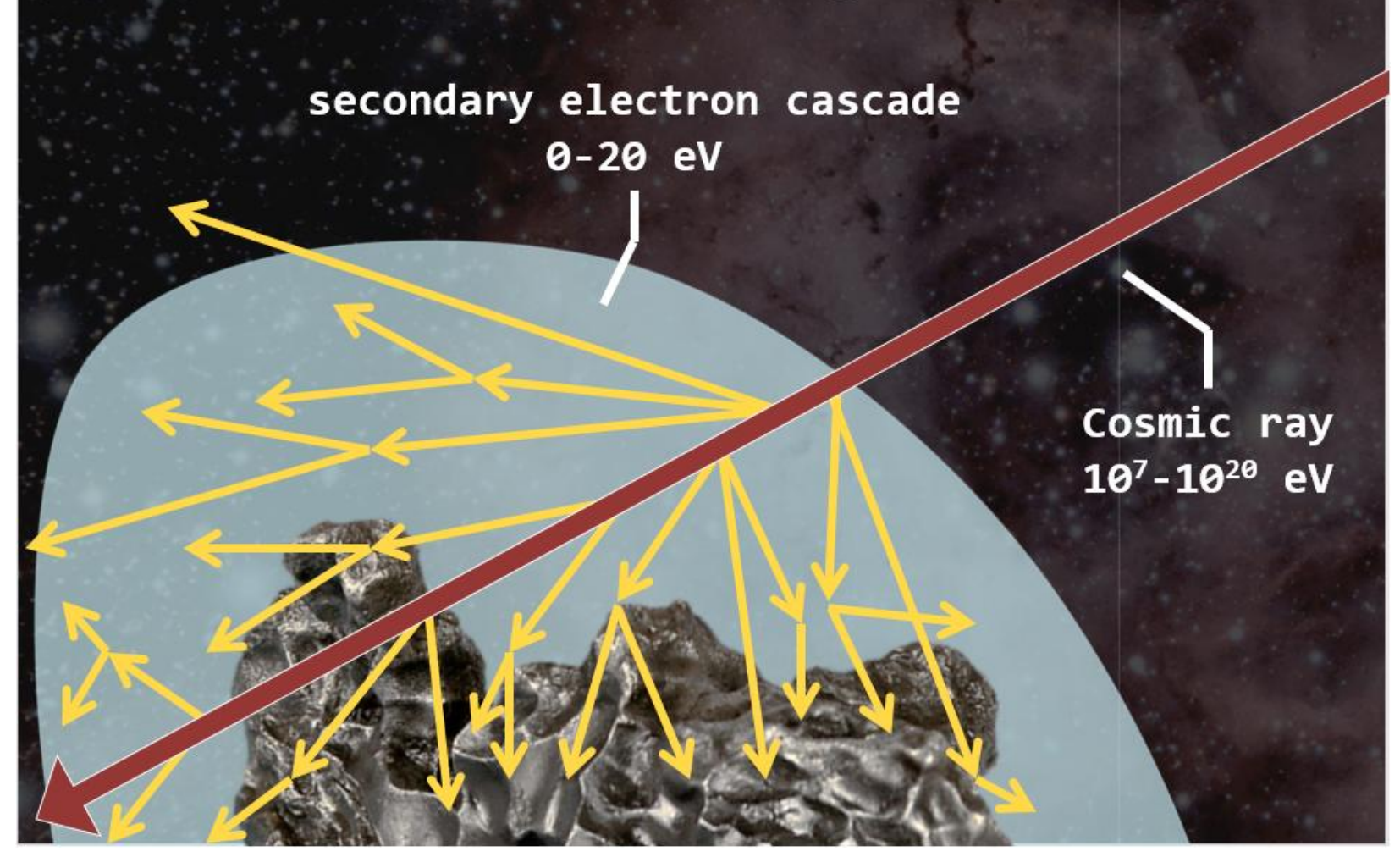

Cosmic ray $10^{7}-10^{2 \theta} \mathrm{eV}$ 